\title{
H-Infinity Design for Automatic Landing System
}

\author{
Serena Cristiana Voicu and Florentin Alin Buţu
}

\begin{abstract}
This study focuses on the H-inf control of aircraft during landing, our aim being also to design a robust control law for a wind shear profile using the $\mathbf{H}$-inf control. This method is based on minimizing a closed loop transfer function norm in order to obtain the stability and robust performance of the system. Some of the design objectives are suggested in other works, but the numerical case study and the numerical implementation of the design procedure are performed and presented in this paper for the first time.
\end{abstract}

Index Terms - Landing, H-inf, control law, wind shear.

\section{INTRODUCTION}

Considering the very low visibility conditions in winter in North-West Europe, Great Britain developed seven decades ago, for the first time, a new system, called automatic landing. At these times, visibility could become as low as a few feet and affected the main UK centres, airports such as Heathrow, Gatwick, Birmingham, Glasgow and European cities such as Paris, Brussels, Amsterdam and Milan. After 1945, two state-owned airline corporations were founded-British European Airways (BEA) and British Overseas Airways Corporation (BOAC). During the immediate post-war period, BEA suffered a several number of accidents during landing in conditions of low visibility. That became a very important problem for the pilots: how they could land safely in such conditions. The development of an automatic landing system was international, but the basis of this creation was developed by the Blind Landing Experimental Unit-BLEU. The design of such a system is based on radio navigation, called today Instrument Landing System (ILS) accepted as a standard system in 1947. It provides the vertical and horizontal guidance for a safe landing in conditions of reduced visibility consisting of a number of phases. This kind of system is used to descend along the runway at a glide path of $3^{\circ}$ maintaining the aircraft onto the runway centre-line. At $30 \mathrm{ft}(9 \mathrm{~m})$ above the ground, the aircraft pitches up to reduce the vertical speed [1]-[4]. There are various categories according to the conditions of visibility which determine the minimum altitude at which the system may be used. The landing categories depend on the decision height $(\mathrm{DH})$, which means the minimum altitude, and the runway

Manuscript received January 16, 2017; revised May 1, 2017. This work is supported by grant no. 89/1.10.2015 of the Romanian National Authority for Scientific Research and Innovation, CNCS-UEFISCDI, code PN-II-RU-TE-2014-4-0849.

Serena Cristian Voicu and Florentin Alin Buţu are with Faculty of Aerospace Engineering, University "Politehnica" of Bucharest, Bucharest, Romania (e-mail: voicuserena@yahoo.com, florentinalin@yahoo.com). visual range (RVR). The first category, CAT I, specifies a decision height not lower than $200 \mathrm{ft}(60 \mathrm{~m})$ and a minimal visibility $1800 \mathrm{ft}(550 \mathrm{~m})$. If the runway visibility range is smaller than $1800 \mathrm{ft}(550 \mathrm{~m})$ or the runway is not in sight, the pilot is required to execute a missed approach and repeat the landing or divert to an alternative. For CAT II, the minimal decision height is $100 \mathrm{ft}(30 \mathrm{~m})$ and the visibility of the runway is minimum $1200 \mathrm{ft}(360 \mathrm{~m})$. CAT III is divided into three sub-categories: CAT IIIa, CAT IIIb and CAT IIIc. CAT IIIa allows the pilot to take over control at the point of touchdown making an automatic landing. The minimal decision height is lower than $100 \mathrm{ft}(30 \mathrm{~m})$ and the RVR is minimum $700 \mathrm{ft}(215 \mathrm{~m})$. For CAT IIIb, the difference is that the pilot takes over control at some distance after touchdown with DH lower than $50 \mathrm{ft}(15 \mathrm{~m})$ and RVR $150 \mathrm{ft}$ ( $45 \mathrm{~m})$. CAT IIIc provides zero visibility, thus the landing is totally automatic but no system has yet been certified to be able to provide such a performance [5]-[10].

This report brings the formulation of the longitudinal dynamics of a transport class aircraft in conditions of wind shear and turbulence. The components of the wind shear are included in the longitudinal dynamic model. Wind shear refers to a difference in wind velocity over short distances which can rapidly cause a lift variation, hence an altitude variation, of the aircraft [11]-[14]. Wind shear is defined as having two components: horizontal and vertical velocity which can affect the aircraft simultaneously. The vertical shear has the most dangerous effect: the change in velocity determines a change in the indicated speed and in thrust requirements. Supposing that the aircraft is using an instrument landing system approach and it is affected by wind shear, it causes a loss of lift. The aircraft is descending and dropping below the glide path. The pilot has to recover this loss of airspeed increasing pitch and power. A very important factor is whether there is enough altitude to correct the path. Therefore, if there is sufficient altitude to recover the desired airspeed and glide path, the pilot is easily able to land safely [2]. The most dangerous forms of wind shear are the microburst and downburst, which have a very short duration of about 3-5 minutes. When wind shear is present at ground level, at the altitude of the runways, it can produce serious disasters changing its airspeed during landing. A serious aircraft landing accident, also presented in [8], occurred on approach to John F. Kennedy International Airport in June 1975 when 113 of the 124 passengers died, accident caused by a microburst.The most recent air catastrophe was also caused by microburst and lack of information on hazardous consequences of the wind shear killing 136 passengers on board on Dallas/Fort Worth International Airport in August 1985. This study focuses on treating this problem using an advanced technique to achieve the proper results. The problem is to design a robust control law for a wind shear profile using $\mathrm{H}$ infinity theory. This method is based on minimizing a closed loop transfer function norm to 
obtain the stability and robust performance of the system. Some of the design objectives are suggested in [7] but the numerical case study and numerical implementation of the design procedure are performed and firstly presented in this paper.

The paper is structured in several sections. The second part of this work contains preliminaries and problem formulation. After presenting the generalized system design model, the third part will describe design methods with a case study - the Boeing 737 model- in the last section.

\section{PRELIMINARIES AND PROBLEM ForMULATION}

The general system model used for this problem formulation contains the so called generalized plant $\mathrm{T}(\mathrm{s})$ and the controller $\mathrm{K}(\mathrm{s})$. $\mathrm{T}(\mathrm{s})$ includes both the dynamics of the aircraft and the wind shear equations.

\section{A. Plant Model}

The glideslope tracking problem was formulated for Boeing 737 aircraft, a similar one to which was involved in the accident on approach to John F. Kennedy International Airport in 1975. The following conditions are used for the mathematical model proposed.
glideslope:
3 degrees
airspeed:
125 knots $(211.593 \mathrm{ft} / \mathrm{sec})$

flaps: 40 degrees

weight: $80,000 \mathrm{lbs}$

centre of gravity:

$25 \%$ of the MAC (mean aerodynamic chord)

The state equations for the plant model, $\mathrm{P}(\mathrm{s})$ are defined below:

$$
\begin{gathered}
\dot{x}=A x+B \mathrm{u}+B_{w} d_{w}, \\
y=C x+D \mathrm{u}+D_{w} d_{w}+\eta,
\end{gathered}
$$

where:

$$
\begin{aligned}
& x=\left[\begin{array}{lllllll}
u & w & q & \theta & u_{w} & w_{w} & h
\end{array}\right]^{T}, \\
& \mathrm{u}=\left[\begin{array}{ll}
\delta_{e} & \delta_{T}
\end{array}\right]^{T}, \\
& d_{w}=\left[\begin{array}{ll}
u_{w g} & w_{w g}
\end{array}\right]^{T}, \\
& y=\left[\begin{array}{lllll}
V_{a} & q & \theta & h & \dot{h}
\end{array}\right]^{T}, \\
& \eta=\left[\begin{array}{lllll}
\eta_{V_{a}} & \eta_{q} & \eta_{\theta} & \eta_{h} & \eta_{\dot{h}}
\end{array}\right]^{T} .
\end{aligned}
$$

The first four states of vector $x$ are the longitudinal states ( $u$ and $\mathrm{w}$ represent the longitudinal velocity in the $x$ and $z$ directions of the body axes, pitch rate $(q)$, and pitch attitude $(\theta), u_{w}$ and $w_{w}$ are the longitudinal and vertical components of the wind velocity and $h$ is the altitude of the aircraft along the glideslope. The two components of the wind vary linearly with respect to decreasing altitude by the horizontal and vertical gradients $U_{z}$ and $W_{z}$. The wind turbulence model is represented by $d_{w}$ with its horizontal and vertical wind gust components: $u_{w g}$ and $w_{w g}$. The vector u consists of the elevator deflection $\left(\delta_{e}\right)$ and the engine thrust $\left(\delta_{T}\right)$. The measurement vector, $\mathrm{y}$, has five components: the airspeed $\left(V_{a}\right)$, pitch rate $(q)$, pitch attitude $(\theta)$, the aircraft altitude $(h)$, and altitude rate $(\dot{h})$. The vector $\eta$ is the measurement noise.

\section{B. Actuator Model}

The state model associated with the actuator dynamics is represented as follows:

$$
\begin{gathered}
\dot{x}_{A}=A_{A} x_{A}+B_{A} \mathrm{u}_{\mathrm{c}}, \\
u_{c}=C_{A} x_{A},
\end{gathered}
$$

where:

$$
\begin{gathered}
x_{A}=\left[\begin{array}{lll}
\delta_{e} & \delta_{t h} & \delta_{T}
\end{array}\right], \\
u_{c}=\left[\begin{array}{ll}
\delta_{e_{c}} & \delta_{t h_{c}}
\end{array}\right] .
\end{gathered}
$$

The vector $x_{A}$ consists of the elevator dynamics, throttle dynamics and engine thrust dynamics. The vector $u_{c}$ includes the elevator position command $\left(\delta_{e_{c}}\right)$ and the throttle position command $\left(\delta_{t h_{c}}\right)$.

\section{Performance Weighting Model}

The performance weighting is applied to the three individual error signals and its state equations are defined as follows:

$$
\begin{aligned}
& \dot{x}_{w p}=A_{w p} x_{w p}+B_{w p} \mathrm{z}_{e}, \\
& z_{e}^{\prime}=C_{w p} x_{w p}+D_{w p} z_{e}
\end{aligned}
$$

$z_{e}$ can be written as:

$$
z_{e}=C_{z e} x I_{3} r_{c}
$$

where:

$$
r_{c}=\left[\begin{array}{lll}
h_{c} & V_{a c} & \theta_{c}
\end{array}\right]^{T},
$$

$r_{c}$ is the command vector.

\section{Wind Turbulence Model}

The state model associated with the wind turbulence through the longitudinal and vertical gust components, $u_{w g}$ and $w_{w g}$, which represent the vector $d_{w}$, can be written as follows:

$$
\begin{gathered}
\dot{x}_{w g}=A_{w g} x_{w g}+B_{w p} n_{w g}, \\
y_{w g}=C_{w g} x_{w g},
\end{gathered}
$$

where:

$$
\begin{gathered}
x_{w g}=\left[\begin{array}{ccc}
u_{w g} & w_{w g_{1}} & w_{w g_{2}}
\end{array}\right]^{T}, \\
n_{w g}=\left[\begin{array}{ll}
n_{u} & n_{w}
\end{array}\right]^{T}, \\
y_{w g}=\left[\begin{array}{ll}
u_{w g} & w_{w g}
\end{array}\right]^{T} .
\end{gathered}
$$

Although there is no dynamic weighting for the measurement noise vector, it could be explained by defining an appropriate high-frequency noise spectrum.

\section{DESIGN METHOD}

The objective of this study is to track the glideslope, despite the conditions of wind shear and turbulence, during landing. This method allows the minimizing of the error in the altitude along the glideslope $(h)$, the airspeed $\left(V_{a}\right)$, and the pitch attitude 
$(\theta)$, thus a minimizing of the effects of wind shear on the aircraft dynamics.

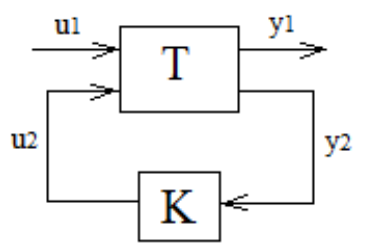

Fig. 1. Block diagram of general system.

The vector of the exogenous inputs, $\mathrm{u}_{1}$, consists of the command signal vector $\left(r_{c}\right)$, the measurement noise vector $(\eta)$, and disturbances vector $\left(n_{w g}\right)$. The vector $r_{c}$ includes the commanded altitude, airspeed and pitch attitude. The components of the vector $n_{w g}$ are white, unit variance noise signals. The vector $y_{1}$ contains the controlled output variables (e.g. tracking errors). These two vectors can be written as follows

$$
u_{1}=\left[\begin{array}{c}
n_{w g} \\
r_{c} \\
\eta
\end{array}\right]=\left[\begin{array}{c}
n_{u} \\
n_{w} \\
h_{c} \\
V_{a c} \\
\theta_{c} \\
\eta_{V_{a}} \\
\eta_{q} \\
\eta_{\theta} \\
\eta_{h} \\
\eta_{h}
\end{array}\right], y_{1}=\left[\begin{array}{c}
\varepsilon_{h} \\
\varepsilon_{V_{a}} \\
\varepsilon_{\theta} \\
\delta_{e} \\
\delta_{t h} \\
\dot{\delta}_{e} \\
\dot{\delta}_{t h}
\end{array}\right] .
$$

The vector $u_{2}$ is the control input vector and consists of errors associated with altitude, airspeed and pitch attitude, elevator and throttle positions and rates. The vector $y_{2}$ represents the measurement vector. The vectors are written as below:

$$
u_{2}=u_{c}=\left\lfloor\begin{array}{c}
\delta_{e_{c}} \\
\delta_{t h_{c}}
\end{array}\right\rfloor, y_{2}=\left[\begin{array}{lllll}
V_{a} & q & \theta & h & \dot{h}
\end{array}\right]^{T} .
$$

The generalized system depicted in Fig. 1 has the state space representation:

$$
\begin{aligned}
& \dot{x}_{G}=A_{G} x_{G}+B_{1} u_{1}+B_{2} u_{2}, \\
& y_{1}=C_{1} x_{G}+D_{11} u_{1}+D_{12} u_{2}, \\
& y_{2}=C_{2} x_{G}+D_{21} u_{1}+D_{22} u_{2} .
\end{aligned}
$$

where

$$
\begin{gathered}
x_{G}^{T}=\left[\begin{array}{llll}
x & x_{A} & x_{w g} & x_{w p}
\end{array}\right]^{T}=\left[\begin{array}{lllllll}
u & w & q & \theta & u_{w} & w_{w} & h
\end{array}\right]^{T}, \\
\dot{x}=A x+B C_{A} x_{A}+B_{w} C_{w g} x_{w g} \\
\dot{x}_{A}=A_{A} x_{A}+B_{A} u_{c} \\
\dot{x}_{w g}=A_{w g} x_{w g}+B_{w g} h_{w g} \\
\dot{x}_{w p}=A_{w p} x_{w p}+B_{w p} C_{z_{e}} x-B_{w p} r_{c}
\end{gathered}
$$

The vectors $x, x_{A}$ and $x_{w g}$ are expressed in equations (3), (10) and (18), respectively. The vector $x_{w p}$ contains the states associated with the performance weighting functions for the errors which are contained in the vector y1. The elements of the vectors $n_{w g}$ and $r_{c}$ are defined in equations (19) and (15).
The above system can be written in matrix form as follows:

$$
\begin{aligned}
{\left[\begin{array}{c}
\dot{x} \\
\dot{x}_{A} \\
\dot{x}_{w g} \\
\dot{x}_{w p}
\end{array}\right]=} & {\left[\begin{array}{cccc}
A & B C_{A} & B_{w} C_{w g} & 0 \\
0 & A_{A} & 0 & 0 \\
0 & 0 & A_{w g} & 0 \\
B_{w p} C_{z_{e}} & 0 & 0 & A_{w p}
\end{array}\right]\left[\begin{array}{c}
x \\
x_{A} \\
x_{w g} \\
x_{w p}
\end{array}\right]+} \\
& +\left[\begin{array}{cc}
0 & 0 \\
0 & 0 \\
B_{w g} & 0 \\
0 & -B_{w p}
\end{array}\right]\left[\begin{array}{c}
n_{w g} \\
r_{c} \\
\eta
\end{array}\right]+\left[\begin{array}{c}
0 \\
B_{A} \\
0 \\
0
\end{array}\right]\left[\begin{array}{c}
\delta_{e_{c}} \\
\delta_{t_{c}}
\end{array}\right],
\end{aligned}
$$

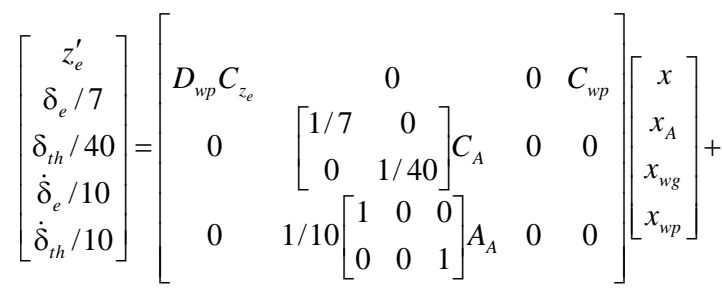

$$
+\left[\begin{array}{ccc}
0 & -D_{w p} & 0 \\
0 & 0 & 0 \\
0 & 0 & 0
\end{array}\right]\left[\begin{array}{c}
n_{w g} \\
r_{c} \\
\eta
\end{array}\right]+\left[\begin{array}{c}
0 \\
1 / 10\left[\begin{array}{ccc}
1 & 0 & 0 \\
0 & 0 & 1
\end{array}\right] B_{A}
\end{array}\right]\left[\begin{array}{c}
\delta_{e_{c}} \\
\delta_{t h_{c}}
\end{array}\right],
$$

$\left[\begin{array}{c}V_{a} \\ q \\ \theta \\ h \\ \dot{h}\end{array}\right]=\left[\begin{array}{llll}C & 0 & 0 & 0\end{array}\right]\left[\begin{array}{c}x \\ x_{A} \\ x_{w g} \\ x_{w p}\end{array}\right]+\left[\begin{array}{ll}0 & I_{5}\end{array}\right]\left[\begin{array}{c}n_{w g} \\ r_{c} \\ \eta\end{array}\right]+\left[\begin{array}{ll}0 & 0\end{array}\right]\left[\begin{array}{c}\delta_{e_{c}} \\ \delta_{t h_{c}}\end{array}\right]$.

The matrix of the generalized system has the following form:

$$
T=\left\lfloor\begin{array}{ccc}
A_{G} & B_{1} & B_{2} \\
C_{1} & D_{11} & D_{12} \\
C_{2} & D_{21} & D_{22}
\end{array}\right\rfloor .
$$

The matrices $A_{w}$ and $B_{w}$ for the plant model are very important because they contain the wind shear gradients and they are given as follows:

$$
\begin{gathered}
A=\left[\begin{array}{ccc}
A_{a a} & A_{a w} & 0 \\
A_{w a} & A_{w w} & 0 \\
A_{h} & 0 & 0
\end{array}\right], \\
B_{w}=\left\lfloor\begin{array}{cc}
-a_{11} & -a_{12} \\
\vdots & \vdots \\
-a_{71} & -a_{72}
\end{array}\right\rfloor,
\end{gathered}
$$

where:

$$
\begin{aligned}
A_{a a}= & \left.\begin{array}{llll}
0.4179 E-01 & 0.98126 E-01 & 0.49565 E+01 & 0.32155 E+02 \\
0.29344 E+00 & 0.76071 E+00 & 0.21178 E+03 & 0.96749 E+00 \\
0.30862 E-03 & 0.49839 E-02 & -0.52858 E+00 & -0.37077 E-03 \\
0.00000 E+00 & 0.00000 E+00 & 0.10000 E+01 & 0.00000 E+00
\end{array}\right] \\
A_{a w} & =\left[\begin{array}{lll}
0.41790 E-01 & -0.98126 E-01 \\
0.29344 E+00 & 0.76071 E+00 \\
0.30862 E-03 & 0.49839 E-02 \\
0.00000 E+00 & 0.00000 E+00
\end{array} \mid\right. \\
A_{w a} & =\left[\begin{array}{llll}
a_{w 11} & a_{w 12} & a_{w 13} & a_{w 14} \\
a_{w 21} & a_{w 22} & a_{w 23} & a_{w 24}
\end{array} \mid\right.
\end{aligned}
$$


$a_{w 11}=0.028944 U_{z}+0.0008384 W_{z}, a_{w 12}=0.999 U_{z}+0.02894 W_{z}$,

$a_{w 13}=0$,

$a_{w 14}=-210.89 U_{z}-17.188 W_{z}, a_{w 21}=-0.0008384 U_{z}+0.0289 W_{z}$,

$a_{w 22}=-0.028944 U_{z}+0.9991 W_{z}, a_{w 23}=0, a_{w 24}=17.188-210.89 W_{z}$,

$A_{w w}=\left[\begin{array}{cc}a_{w 15} & 0 \\ 0 & a_{w 26}\end{array}\right], a_{w 15}=a_{w 16}=-0.01$,

$A_{h}=\left[\begin{array}{llll}-0.02895 & -0.999 & 0 & 211.3\end{array}\right]$.

The vertical and horizontal wind shear gradients $U_{z}$ and $W_{z}$ vary as follows: $-0.25 \leq \mathrm{Uz} \leq 0.39$ (Increasing Tailwind); $-0.47 \leq \mathrm{Wz} \leq 0.33$ (Increasing Downdraft). The actuator state model, which is expressed by the equations (8) and (9), has the following matrix:

$A_{A}=\left\lfloor\begin{array}{ccc}-\omega_{\delta_{e}} & 0 & 0 \\ 0 & -\omega \delta_{\text {th }} & 0 \\ 0 & K_{\text {eng }} \omega \delta_{\text {eng }} & -\omega \delta_{\text {eng }}\end{array}\right\rfloor, B_{A}=\left[\begin{array}{cc}\omega_{\delta_{e}} & 0 \\ 0 & \omega \delta_{\text {th }} \\ 0 & 0\end{array}\right\rfloor, C_{A}=\left[\begin{array}{lll}1 & 0 & 0 \\ 0 & 0 & 1\end{array}\right]$

The performance aim was expressed relative to the controlled vector $\left(y_{1}\right)$, which contains controlled variables and tracking errors. The requirements were formulated relative to the following time response characteristics: Time Constant: $1.0 \mathrm{sec}$; Steady-State Error: 0.1\%, Step Response Overshoot: $10 \%$. The requirements defined above are used to express a performance weighting function $\left(W_{p}\right)$, as below:

$$
W_{p}=\frac{K_{p}\left(s+\omega_{1}\right)}{s+\omega_{2}}
$$

The weighted control position and rate signals are based on the actuator position and rate limits which are written below:

Elevator Position:

$$
\delta_{e} \leq \pm 10 \mathrm{deg}
$$

$$
\begin{aligned}
& \text { Trim }=3.2 \mathrm{deg} \\
& \left|\delta_{e_{\text {max }}}\right|=7 \mathrm{deg} \\
& 10 \leq \delta_{\text {th }} \leq 60 \mathrm{deg} \\
& \text { Trim }=17.6 \mathrm{deg} \\
& \left|\delta_{t_{\text {max }}}\right|=40 \mathrm{deg}
\end{aligned}
$$

Elevator Rate Limit: $10 \mathrm{deg} / \mathrm{sec}$

Throttle Rate Limit: $10 \mathrm{deg} / \mathrm{sec}$

The weightings of the control positions and rates can be selected to

be:

$W_{\delta_{e}}=1 / 7, W_{\delta_{b h}}=1 / 40, W_{\delta_{e}(\text { rate })}=1 / 10, W_{\delta_{\delta_{h}}(\text { rate })}=1 / 10$. The matrices which are included in the performance weighting model are defined as follows:

$$
\begin{aligned}
A_{w p} & =\left[\begin{array}{ccc}
-0.001 & 0 & 0 \\
0 & -0.001 & 0 \\
0 & 0 & -0.001
\end{array}\right], B_{w p}=\left[\begin{array}{ccc}
0.1 & 0 & 0 \\
0 & 0.1 & 0 \\
0 & 0 & 0.1
\end{array}\right], \\
C_{w p} & =\left[\begin{array}{ccc}
9.99 & 0 & 0 \\
0 & 9.99 & 0 \\
0 & 0 & 9.99
\end{array}\right], D_{w p}=\left[\begin{array}{ccc}
0.1 & 0 & 0 \\
0 & 0.1 & 0 \\
0 & 0 & 0.1
\end{array}\right] .
\end{aligned}
$$

The wind disturbance weighting matrix, $W_{g}(\mathrm{~s})$, is associated with the wind gust disturbance vector, $d_{w}$, defined in equation (5). The Dryden turbulence model provides low pass turbulence weighting functions for the longitudinal and vertical wind gust components, $u_{w g}$ and $w_{w g}$. The Dryden wind turbulence model uses the linear velocity and angular components of gust as variable. The Dryden wind turbulence model is defined by power spectral densities and is used to add turbulence to the model by passing band-limited white noise through appropriate forming filters. According to the Military Specification MIL-F-8785C and Military Handbook MIL-HDBK-1797, turbulence is a stochastic process. For an aircraft flying at airspeed $V_{a}$ in a turbulent atmosphere, the transfer functions are expressed as below; the model is represented in Fig. 2.

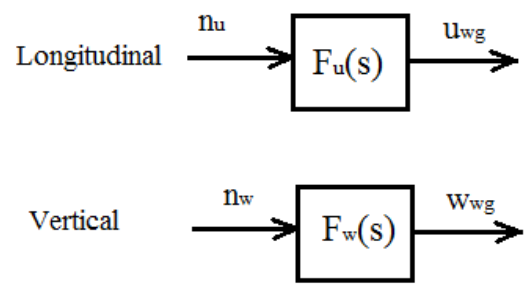

Fig. 2. Dryden turbulence model.

$$
F_{u}(s)=\sigma_{u} \sqrt{\frac{L_{u}}{\pi V_{a}}}\left\lfloor\frac{1}{1+\frac{L_{u}}{V_{a}} s}\right\rfloor \quad F_{w}(s)=\sigma_{w} \sqrt{\frac{L_{w}}{2 \pi V_{a}}}\left\lfloor\frac{1+\sqrt{3}\left(\frac{L_{w}}{V_{a}}\right) s}{\left(1+\frac{L_{w}}{V_{a}} s\right)^{2}}\right\rfloor
$$

The wind disturbance weighting matrix, $W_{g}(\mathrm{~s})$, is determined by the expression: $W_{g}(\mathrm{~s})=\operatorname{diag}\left(F_{u}(\mathrm{~s}), F_{w}(\mathrm{~s})\right)$. The state equations of the disturbance weighting are defined in equations (16) and (17). The matrices $A_{w g}, B_{w g}$ and $C_{w g}$ are defined below:

$$
\begin{aligned}
& A_{w_{s}}=\left[\begin{array}{ccc}
-\frac{V_{a}}{L_{u}} & 0 & 0 \\
0 & -\frac{V_{a}}{L_{w}} & 0 \\
0 & \sigma_{w} \frac{1}{\sqrt{L_{w}}} \sqrt{\frac{3 V_{a}}{2 \pi}} & -\frac{V_{a}}{L_{w}}
\end{array}\right\rfloor, B_{w_{s}}=\left[\begin{array}{cc}
\sigma_{u} \frac{1}{\sqrt{L_{u}}} \sqrt{\frac{V_{a}}{\pi}} & 0 \\
0 & -V_{a} \frac{1}{L_{w}}\left(1-\frac{1}{\sqrt{3}}\right) \\
0 & \sigma_{w} \frac{1}{\sqrt{L_{w}}} \sqrt{\frac{3 V_{a}}{2 \pi}}
\end{array}\right], \\
& C_{w_{s}}=\left\lfloor\begin{array}{lll}
1 & 0 & 0 \\
0 & 0 & 1
\end{array}\right\rfloor .
\end{aligned}
$$

\section{A CASE StUdy - Boeing 737 Model}

Introducing the parameters for the selected aircraft model, the matrices included in the state equations for the plant model are written below. The numerical values used in this case study are given in [7]. For the weighting transfer matrix $\mathrm{W}_{\mathrm{P}}$ chosen in this paper is $W_{P} s=0.1 s+2000 / s+0.001$ which frequency response is presented in Fig. 3. The values of vertical and horizontal wind shear gradients $U_{z}$ and $W_{\mathrm{z}}$ are chosen $0.1 \mathrm{ft} / \mathrm{sec}$ for both the gradients. These values correspond to and increasing tailwind simultaneous with an increasing downdraft. The used numerical values in simulations are:

$A=\left[\begin{array}{ccccccc}-0.0042 & 0.0981 & -4.9565 & -32.155 & 0.0418 & -0.0981 & 0 \\ -0.2934 & -0.7607 & 211.78 & 0.9675 & 0.2934 & 0.7607 & 0 \\ -0.0003 & -0.005 & -0.5286 & -370.77 & 0.0003 & 0.005 & 0 \\ 0 & 0 & 1 & 0 & 0 & 0 & 0 \\ 0.003 & 0.1028 & 0 & -22.8078 & -0.001 & 0 & 0 \\ 0.0028 & 0.097 & 0 & -19.3702 & 0 & -0.001 & 0 \\ -0.029 & -0.9996 & 0 & 211.3 & 0 & 0 & 0\end{array}\right\rfloor$




$$
B=\left[\begin{array}{cc}
0.004 & 0.0004 \\
-0.1725 & 0 \\
-0.021 & 0 \\
0 & 0 \\
0 & 0 \\
0 & 0 \\
0 & 0
\end{array} \mid\right.
$$

$C=\left[\begin{array}{ccccccc}0.9997 & 0.0234 & 0 & 0 & -0.9997 & -0.0234 & 0 \\ 0 & 0 & 1 & 0 & 0 & 0 & 0 \\ 0 & 0 & 0 & 1 & 0 & 0 & 0 \\ 0 & 0 & 0 & 0 & 0 & 0 & 1 \\ -0.028956 & -0.99958 & 0 & 211.3 & 0 & 0 & 0\end{array}\right\rfloor$

For the actuator model the following values are used (see [7])

$$
\begin{aligned}
& \omega_{\delta_{\mathrm{e}}}=16 \mathrm{rad} / \mathrm{s}, \omega_{\delta_{\text {th }}}=10 \mathrm{rad} / \mathrm{s}, \omega_{\delta_{\text {eng }}}=0.5 \mathrm{rad} / \mathrm{s}, \\
& K_{\text {eng }}=5961 \mathrm{bs} / \mathrm{deg}, V_{a}=211.53 \mathrm{ft} / \mathrm{s} .
\end{aligned}
$$

The matrices $A_{A}, B_{A}$ are defined as follows:

$$
A_{A}=\left[\begin{array}{ccc}
-16 & 0 & 0 \\
0 & -10 & 0 \\
0 & 298 & -0.5
\end{array}\right] \quad B_{A}=\left[\begin{array}{cc}
16 & 0 \\
0 & 10 \\
0 & 0
\end{array}\right] .
$$

The matrices for the performance weighting model are defined in equations (33). For the Dryden disturbance models (34)-(36), the following turbulence specifications for the wind profile and for the altitude $h=200.0656 f t$ have been used $\quad L_{u}=306.595 \mathrm{ft}, L_{w}=106.037 \mathrm{ft}, \sigma_{u}=7.477 \mathrm{ft} / \mathrm{s}$, $\sigma_{w}=7.342 \mathrm{ft} / \mathrm{s}$.

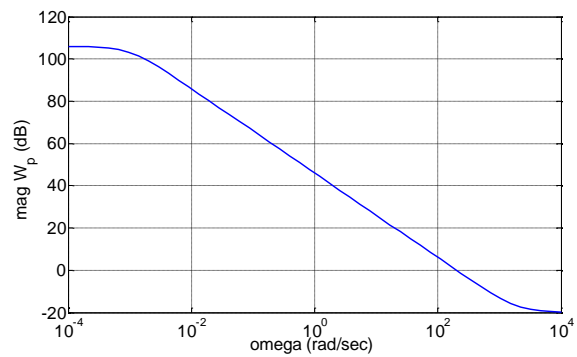

Fig. 3. Magnitude of the weighting function $W p$.

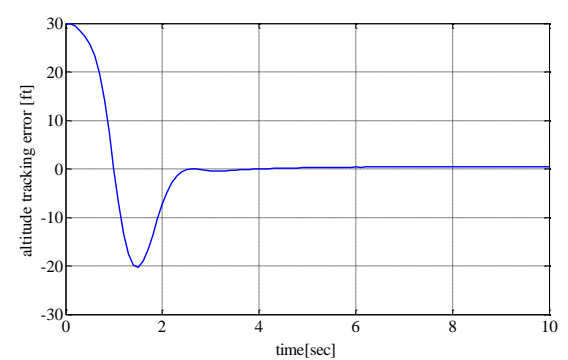

Fig. 4. Time response of the altitude tracking error.

In Figs. 3-5 there are presented the magnitude of the weighting function $W_{p}$, the time response of the altitude tracking error, and the pitch angle tracking error time response. These time responses have been obtained for an initial altitude deviation with respect to the ideal glide slope of $30 \mathrm{ft}$ and an initial deviation of the pitch angle of 10 degrees, respectively. In these numerical simulations a value of $0.1 \mathrm{ft} / \mathrm{sec}$ for the wind shear gradients $U_{z}$ and $W_{z}$ have been considered, corresponding to increasing tailwind and to increasing downdraft.

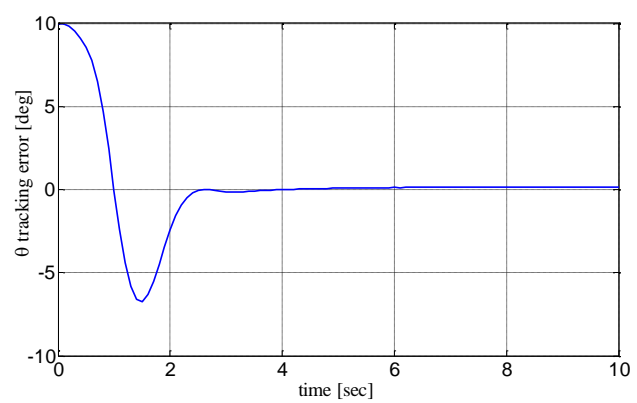

Fig. 5. The pitch angle tracking error time response.

\section{CONCLUSION}

This study has presented the problem formulation of a glideslope tracking through severe wind shear and gusts conditions. The parameters were based on the reconstruction of the wind profile that caused the landing accident at Kennedy International Airport in 1975. The Dryden turbulence model has provided weighting information and the wind gusts were included as disturbance inputs to the plant. Using the $\mathrm{H}$ infinity theory, stability and robust performance has been obtained. This system has been able to allow the aircraft to track the glideslope, despite severe conditions, during the landing. This advanced theory allows minimizing of the effects of wind shear and turbulence on the aircraft dynamics.

\section{REFERENCES}

[1] R. Lungu and M. Lungu, "Application of $\mathrm{H}_{2} / \mathrm{H}_{\infty}$ and dynamic inversion techniques to aircraft landing control," Aerospace Science and Technology, vol. 46, pp. 146-158, 2015.

[2] Wind Shear, publication of Federal Aviation Administration.

[3] M. Lungu and R. Lungu, "Automatic control of aircraft lateral-directional Motion during landing using neural networks and radio-technical subsystems," Neurocomputing Journal, vol. 171, pp. 471-481, 2016.

[4] D. McLean, Automatic Flight Control Systems, Prentice Hall Publisher, 1990 .

[5] B. Etkin and L.D. Reid, Dynamics of Flight. Stability and Control, 3rd Edition, Willey Publisher, 1996.

[6] J. Blakelock, Automatic Control of Aircraft and Missiles, 2nd Edition, 1991.

[7] C. M. Belcastro and B. C. Chang, "A problem formulation for glideslope tracking in wind shear using advanced robust control techniques," NASA Technical Reports, 1992, pp. 1-21.

[8] Committee on Low-Altitude Wind Shear and Its Hazard to Aviation; Aeronautics and Space Engineering Board; Board on Atmospheric Sciences and Climate; Commission on Engineering and Technical Systems; Division on Engineering and Physical Sciences, National Research Council, 1983.

[9] J. Dieudonne, "Comments on a proposed standard wind hazard environment and its use in real-time aircraft simulations," Report: AIAA PAPER 79-0324, 1979.

[10] J. J. Shrager, The analysis of NTSB large fixed-wing aircraft accident/incident reports for the potential presence of low-level wind shear, FAA-DD-77-169. Federal Aviation Administration, December 1977.

[11] R. Lungu, M. Lungu, and T. L. Grigorie,"ALSs with conventional and fuzzy controllers considering wind shears and gyro errors," Journal of Aerospace Engineering, vol. 26, no. 4, pp. 794-813, 2013

[12] R. Lungu, M., Lungu, and T. L. Grigorie, "Automatic control of aircraft in longitudinal plane during landing," IEEE Transactions on Aerospace, vol. 49, no. 2, pp. 1338-1350, 2013.

[13] D. C. McFarlane and K. Glover, "Robust controller design using normalized coprime factor plant description," Springer-Verlag New 
York, Inc. Secaucus, USA, 1989.

[14] M. Cook, Flight Dynamics Principles, Elsevier Aerospace Engineering Series, 1997.

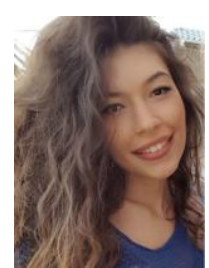

Serena-Cristiana Voicu received a B.Sc. degree in avionics engineering from the University Politehnica of Bucharest in 2016. She is currently a M. Student at Avionics and Air Navigation at University Politehnica of Bucharest. She works as a researcher at National Institute for Aerospace Research "Elie Carfoli" in Bucharest.

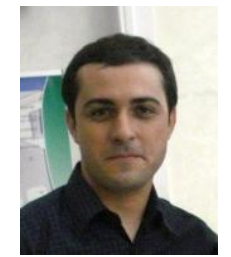

Florentin-Alin Butu received a B.Sc degree in systems engineering from the University of Craiova in 2009 and M.Sc. degree in the information technology in software engineering from the University of Craiova in 2011. He worked as a software engineer in various projects involving developing and testing for critical systems using DO178B standard, numerical engineering software using MATLAB/Simulink, command \& control systems and spaceflight dynamics. He is currently a $\mathrm{Ph} . \mathrm{D}$ student at University POLITEHNICA of Bucharest and his research area is automatic control of spacecraft trajectory and attitude. 